\title{
CLIMA ORGANIZACIONAL EN OCHO EMPRESAS DE AREQUIPA
}

\section{ORGANIZATIONAL CLIMATE AT EIGHT ENTERPRISES FROM AREQUIPA}

Walter L. Arias Gallegos

Universidad Católica San Pablo, Arequipa, Perú

\section{Resumen:}

En esta investigación se describen los perfiles del clima organizacional de ocho empresas de Arequipa. Para ello se aplicó el Perfil Organizacional de Likert (POL) a 352 trabajadores ( 268 varones y 84 mujeres) de entre 20 y 67 años de edad. Se encontró que las dimensiones más desarrolladas del clima organizacional son la claridad $(19,53)$ y la responsabilidad $(17,95)$; mientras que las más deficitarias son el reconocimiento $(12,67)$ y la flexibilidad $(16,94)$. En general, el nivel de clima organizacional de las ocho empresas es moderado alto $(16,80)$. Se concluye que en las empresas evaluadas, los trabajadores perciben que se les exige un desempeño orientado a la calidad y el perfeccionamiento con autonomía en el trabajo, pero como contraparte, no se les brinda el apoyo ni las recompensas necesarias para cumplir con los objetivos organizacionales. Asimismo, ante este panorama, los trabajadores parecen tomar las relaciones interpersonales con sus compañeros, como fuentes de apoyo social que permiten suplir la falta de apoyo institucional. Se presentan sugerencias para implementar estrategias que reconozcan y recompensen los esfuerzos de los trabajadores, así como mediciones de los riesgos psicosociales relacionados con el clima organizacional.

Palabras clave: Clima organizacional, empresa, psicología organizacional.

Summary: In this research we describe organizational climate profiles of eight enterprises from Arequipa. In order to analize them, we applied the Likert's Organizational Profile to 352 workers (268 male and 84 female) among 20 and 67 years old. We found that highest 
dimentions in organizational climate are clarity $(19,53)$ and responsability $(17,95)$; but the lowest dimentions are rewarding $(12,67)$ and flexibility $(16,94)$. The level of organizational climate at eight enterprises is high moderate $(16,80)$. We conclude that in the enterprises of the sample, workers consider they are pushed to have a high job performance but they don 't recive support or apropiate rewards to reach the objectives in work. Moreover, workers seem to take interpersonal interactions as sources of social support.

Key words: Organizational climate, enterprise, organizational psychology.

\section{Introducción}

La clave que da la pauta en los nuevos tiempos —dice Smith (2006) — son dos cualidades que deben ubicarse en el corazón de las organizaciones, como el motor que impulsa sus logros en la consecución de sus metas. Estas cualidades son la flexibilidad y la especialización. Es necesario que las empresas sean flexibles para adaptarse al cambio permanente; y no menos importante es que promuevan la especialización, para que puedan aprovechar al máximo las capacidades y fortalezas de sus trabajadores y colaboradores.

Estos dos factores deben ser considerados como elementos estratégicos para el desarrollo organizacional. En ese sentido, la orientación estratégica propone que todos los subsistemas de la organización estén alineados con el objetivo estratégico para crear un clima de interés y desarrollar un enfoque orientado al servicio del cliente. De ahí que el concepto de clima organizacional sea hoy, más que nunca, la piedra angular del éxito empresarial. Pero hablar de clima organizacional no es suficiente, porque esta no es una variable aislada. El ambiente laboral está determinado por diversos factores: los de tipo general (aspectos sociales, económicos, legales y tecnológicos), los operativos (cliente, trabajo y proveedores) y los de naturaleza interna (total de fuerzas interactuantes dentro de la organización).

En este contexto, el clima organizacional es una de las variables más estudiadas en el ámbito de la psicología organizacional, y ello se debe quizá a las implicancias que tiene en diversos procesos que tienen lugar en el seno de las empresas. Así, por ejemplo, un clima organizacional positivo favorece la existencia elevada de la motivación de los trabajadores, el rendimiento y la productividad, el compromiso y la lealtad de los empleados (Arias, 2007). Por otro lado, también se ha reportado que el estrés disminuye en la medida en que mejora el clima organizacional (Barrón, Soler y Bongiovanni, 2005). En ese sentido, el clima organizacional está relacionado con diversos estresores ocupacionales, que a su vez dependen de la demandas de la tarea, las demandas del puesto, las demandas interpersonales, la estructura organizacional, el liderazgo organizacional y la etapa de vida en que se encuentra la organización (Atalaya, 2001). 
Sin embargo, hoy en día no cabe duda que una organización con una baja calidad de vida o un clima organizacional deficiente puede dañar la salud mental y física de su colectivo laboral. El clima organizacional positivo favorece el cumplimiento de objetivos. Pero para promover un buen clima organizacional se requiere el fortalecimiento de las actividades de promoción de buena salud en la organización, el apoyo de la gerencia, el control de la presencia y el efecto de riesgos asociados, la priorización de la motivación laboral, etc.; es decir, se requiere manejar un concepto global de desarrollo sostenible, salud y seguridad (Salazar et al., 2009).

Es por todo ello que el clima organizacional, así como otros fenómenos psicosociales de las organizaciones como la motivación, las relaciones humanas, la satisfacción laboral, el liderazgo, la comunicación, etc., constituyen factores esenciales en el desempeño del capital humano, por lo que la valoración pertinente del estado de todos estos fenómenos coadyuvará con la precisa formulación de estrategias y métodos que orienten a las empresas hacia el desarrollo organizacional en la búsqueda de la eficiencia y la efectividad.

Arequipa es una ciudad que ha atravesado por diversos periodos, fructuosos algunos y perjudiciales otros, que han trazado una línea del desarrollo industrial y, más ampliamente, del laboral. Hoy en día surge una preocupación, cada vez más constante: el crecimiento sostenido de las empresas y su posicionamiento en un mercado sumamente complejo como competitivo.
Alcanzar esta meta pasa por la prevención de riesgos psicosociales en el trabajo, y éste, a su vez, por el reconocimiento y la valoración de los mismos. Visto así, el clima organizacional y sus diversos componentes cobran un sentido vital para las organizaciones. Los empresarios arequipeños recién se están dando cuenta de ello y valoran con mayor profundidad los aspectos psicosociales del trabajo. Debe tenerse en cuenta, en ese sentido, que en más de 500 organizaciones, de un 50 a 70 por ciento, el clima organizacional depende de sus líderes (Pérez, Maldonado y Bustamante, 2006).

El presente trabajo de investigación pretende determinar el clima organizacional de diversas organizaciones arequipeñas en el rubro de la producción de bienes y la prestación de servicios, con el objeto de establecer un perfil y conocer los elementos o dimensiones del clima organizacional que se encuentran más desarrolladas o que, por el contrario, presentan niveles por debajo del promedio.

\section{Definición de clima organizacional}

Aunque usualmente se atribuye a Gellerman la introducción del término “clima organizacional" dentro del ámbito empresarial, en 1960 (Bouroncle, 2010), el constructo de clima organizacional fue usado por Lewin, Lippit y White en 1939 (Gómez, 2001). Kurt Lewin propuso una psicología topológica que planteaba la existencia del espacio vital dentro de la que se erige un sistema dinámico entre la persona y su contexto. Esta relación era representada 
por Lewin mediante la siguiente fórmula: $\mathrm{C}=\mathrm{f}(\mathrm{P} \times \mathrm{E})$, donde la conducta $(\mathrm{C})$ es una función de la interacción entre la persona (P) y su espacio vital (E) (Arias, 2005); por tanto, dice Lewin, "el concepto de clima es útil para enlazar los aspectos objetivos de la organización" (citado por Vega et al., 2006).

De modo que podemos decir que el concepto de clima organizacional inicia con Lewin (Salgado, Remeseiro e Iglesias, 1996) y más aún, como una aplicación de los conceptos de la psicología de la forma o gestalt theory, que se ha centrado en la percepción como fenómeno integrador de la experiencia. De hecho, la mayoría de conceptos de clima organizacional, lo conciben como la percepción que tienen los individuos sobre el ambiente interno de su trabajo. Brunet, Schneider, Reichers, Toro, Peiró, Álvarez y muchos otros investigadores, también lo definen de manera similar, tomando como núcleo conceptual la percepción que tienen las personas respecto de su trabajo (Vega et al., 2006). De ahí que el constructo de clima organizacional sea visto como clima psicológico (Lassio, 2003), en la medida en que se constituye de la percepción que cada individuo tiene. Sería entonces el criterio de individualidad el que permite definirlo como clima psicológico, pero cuando dicha percepción es compartida por varios trabajadores se suele hablar de manera más precisa de clima organizacional. En otras palabras, la percepción de una sola persona no nos dice mucho del funcionamiento de una organización, pero si esta es una percepción compartida, su alcance y valor diagnóstico para la organización es trascendente. De esta manera, el clima como realidad psicosocial se nutre de las interacciones entre los individuos y la organización, y es el mediador entre los procesos organizacionales y los comportamientos de los trabajadores (Romero, 2001).

El clima organizacional suele relacionarse, sino hasta confundirse, con otras variables psicológicas como la satisfacción laboral. La satisfacción laboral puede definirse como una respuesta emocional positiva al puesto y que resulta de la evaluación de si el puesto cumple o permite cumplir los valores laborales del individuo (Salgado, Remeseiro e Iglesias, 1996). Por ello, guarda estrecha relación con el clima organizacional, en tanto que también se basa en las valoraciones que tiene el trabajador respecto de su entorno laboral. Por ello, algunos autores utilizan los conceptos de clima organizacional y satisfacción laboral como sinónimos (Lassio, 2003). Salgado, Remeseiro e Iglesias (1996) han encontrado que sólo en el factor de relaciones interpersonales se aprecia una correlación significativa entre clima organizacional y satisfacción laboral, por tanto concluyen que ambos son constructos relacionados pero independientes entre sí.

Otro concepto relacionado con el clima organizacional es el de cultura organizacional. Toro, por ejemplo, indica que el clima organizacional es un constructo complejo y multidimensional relacionado con la cultura organizacional de un modo poco claro, que puede estudiarse como causa y como efecto (Gómez, 2001). Y es que la cultura, al igual que el clima organizacional, abarca un sistema de 
significados compartidos por una gran parte de los miembros de una organización que los distingue de otras (Salazar et al., 2009).

El interés en la cultura organizacional comenzó en 1980 con el libro En busca de la excelencia de Peters y Waterman, y recoge una larga tradición como producto de los estudios antropológicos en la vida empresarial (Gómez, Sánchez y Alonso, 2005). Ahora bien, existen semejanzas entre el clima organizacional y la cultura organizacional que deben mencionarse. Por ejemplo, ambos tratan las formas en que el ambiente cobra sentido para los miembros. Los dos son aprendidos y además son constructos multidimensionales que afectan el comportamiento de los trabajadores. Sin embargo, aunque semejantes, también presentan notables diferencias. Mientras la cultura organizacional abarca los valores y concepciones subyacentes a la conducta del trabajador, el clima organizacional abarca el consenso de las percepciones de los trabajadores. La cultura organizacional presenta una evolución en el tiempo, en tanto que el clima organizacional es instantáneo y ahistórico. La cultura organizacional es un concepto más antropológico y sociológico, mientras que el clima organizacional es un concepto psicológico. Metodológicamente, la cultura organizacional se estudia cualitativamente mediante la observación, y el clima organizacional se estudia cuantitativamente mediante encuestas. Finalmente, la cultura organizacional se fundamenta en la teoría sociocrítica, mientras que el clima organizacional se basa en la teoría de Kurt Lewin (Alcover, 2003).
Más allá de las semejanzas o las diferencias entre el clima y la cultura organizacional existen, empero, relaciones entre ambos constructos. Pero, mientras que para algunos el clima organizacional ejerce influencia en la cultura organizacional (Salazar et al., 2009), para nosotros, es la cultura organizacional la que influye en el clima organizacional, ya que la cultura emana en gran medida de los valores y el liderazgo que ejercen los directivos de las empresas, mientras que el clima es la resultante de dichas manifestaciones, sea que se transmitan descendentemente $u$ horizontalmente.

En resumen, se usa el término clima organizacional para describir la estructura psicológica de la organización (Gómez, 2001). En ese sentido, dado que el clima organizacional se relaciona con la satisfacción laboral, la cultura de la empresa y la motivación de los trabajadores, entre otras variables de corte psicológico, no han faltado autores como Robbins (1999) que dicen que el clima organizacional puede ser entendido como la personalidad de la organización, y razón no le falta ya que el clima organizacional es un concepto molecular y sintético como la personalidad (Bouroncle, 2010). Para Chiavenato (2002, 2009) es el medio interno y la atmosfera de una organización. Sudarsky señala que el clima organizacional es el reflejo de una situación grupal de motivación, la cual obedece a diferentes patrones de comportamiento como consecuencia de las necesidades sociales (Vega et al., 2006). Pace entiende el clima organizacional como un patrón de características organizativas con 
relación a la calidad del ambiente interno de la institución. Campbell, Dunnette, Lawler y Weick describen el clima organizacional como un conjunto de atributos específicos de una organización particular que puedeser deducida de la manera como la organización interactúa con sus miembros y con el medio ambiente. Pritchard y Karasick definen el clima organizacional como la cualidad del ambiente interno de una organización que resulta del comportamiento de los miembros y sirve para interpretar situaciones y orientar las actividades dentro de la organización (Gómez, 2001).

Otros autores han propuesto, además del clima organizacional, la existencia de un clima de comunicación como Daly, Falcione y Damhorst; o un clima de creatividad como Taylor y Ellinson, o un clima de seguridad como el propuesto por Zohar (Guillén, Gala y Velázquez, 200o).

A pesar de las diversas definiciones de clima organizacional, éstas hacen referencia a un conjunto de características que definen una organización y que las distinguen de otras, que son duraderas en el tiempo y que tienen además influencia en la conducta de los individuos (Forehad y Gilmer, 1964, citados por Guillén, Gala y Velázquez, 200o). Sin embargo, dado que algunas definiciones resultan vagas en su sentido más general, se han sistematizado los conceptos y teorías del clima organizacional categorizándolos en tres enfoques claramente diferenciados, que abarcan asimismo una diversidad de dimensiones que componen el clima organizacional, pero que varían según los autores.

\section{Enfoques y elementos/dimensiones del clima organizacional}

En vista de la pluralidad de conceptos sobre el clima organizacional, se han propuesto tres enfoques que lo definen sobre una base común para cada uno de ellos. Desde un enfoque objetivo el clima organizacional es definido como un conjunto de características permanentes, tales como el tamaño de la organización, la estructura organizativa, la complejidad de los sistemas organizacionales, el estilo de liderazgo y las orientaciones de las metas. Este sería el enfoque de Campbell, Dunnette, Lawler y Weick. Desde un enfoque subjetivo se considera que el clima organizacional es la percepción conjunta que tienen los miembros de la organización (Pérez, Maldonado y Bustamante, 2006). Este sería el enfoque de Schneider quien define el clima organizacional como la percepción que cada empleado tiene de las rutinas y recompensas del entorno (Lassio, 2003). Reichers lo define como las percepciones compartidas que los miembros desarrollan en relación con las políticas, prácticas y procedimientos organizacionales, tanto formales como informales. Álvarez también se ubica dentro de este enfoque, pues para él, el clima organizacional es la expresión de las percepciones o interpretaciones que el individuo hace del ambiente interno de la organización en la cual participa (Gómez, 2001).

Puede decirse que los enfoques objetivos ponen énfasis en las características de la organización, mientras que los enfoques subjetivos se centran en las percepciones, 
los valores y creencias que tienen los trabajadores. Los enfoques integradores consideran ambos factores, o sea, los factores personales y las variables organizacionales en función de las metas operativas del sistema. La teoría de Renis Likert pertenece a este tipo de enfoque, y se conoce como teoría de los sistemas de organización (Bouroncle, 2010).

Para Likert, el comportamiento de los subordinados es causado por el comportamiento administrativo y por las condiciones organizacionales que estos perciben, y en parte por sus informaciones, percepciones, valores y capacidades. De modo que se deben diferenciar las variables causales, las intermediarias y las finales. Las primeras son de carácter independiente y determinan el curso de la evolución de las organizaciones así como los resultados que obtienen. Las segundas reflejan el interior de la empresa a través de las motivaciones, las actitudes, los objetivos, el rendimiento, la eficacia de la comunicación y la toma de decisiones. Finalmente, las variables finales son de carácter dependiente y son el resultado de las dos anteriores (Bouroncle, 2010). Todas ellas determinan el clima organizacional.

Likert también propone cuatro tipos de clima organizacional. El autoritario explotador es aquel en el que los directivos de la organización no tienen confianza en sus empleados, y existe una diversidad de demandas a través del castigo y el temor que generan en los trabajadores. En el clima organizacional autoritario paternalista no existe confianza en los trabajadores pero tampoco existen presiones sobre ellos, ya que todo recae sobre los directivos o las cabezas de la organización. El tipo participativo consultivo se caracteriza porque existe confianza en los empleados pero las decisiones se toman desde arriba, por tanto la comunicación es de tipo descendente y la empresa se encuentra burocratizada. Finalmente, en el clima en que se promueve la participación de grupo hay confianza y descentralización en todos los niveles, los directivos y los trabajadores se mueven en función de los objetivos estratégicos (Sandoval, 2004).

Además, Likert indica que existen nueve elementos del clima organizacional: 1) los métodos de mando, 2) las fuerzas motivacionales, 3) los procesos de comunicación, 4) los procesos de influencia, 5) los procesos de toma de decisiones, 6) los procesos de planificación, 7) los procesos de control, 8) los objetivos de rendimiento y 9) el perfeccionamiento (Gómez, 2001). De estos elementos, Likert propone seis dimensiones del clima organizacional: flexibilidad, responsabilidad, estándares, reconocimiento, claridad y espíritu de equipo.

Otros autores, al igual que Likert, han planteado según su criterio los elementos y/o dimensiones de los que se compone el clima organizacional. En el Cuadro 1. se puede apreciar doce autores con sus respectivas propuestas sobre los elementos del clima organizacional que cada uno de ellos sugiere. También puede observarse que los elementos que se encuentran incluidos en la mayoría de propuestas son: relaciones 
interpersonales, recompensa, estructura y cooperación. Sin embargo, las variables del clima organizacional más estudiadas son la estructura, la autonomía, los sistemas de remuneración, la relación con la supervisión y el nivel de resolución de conflictos (Guillén Gala y Velázquez, 200o).

Las relaciones interpersonales son las vinculaciones y relaciones directas que se van formando en el curso de la vida laboral, entre los individuos que piensan y sienten (Portugal, 200o). La estructura organizacional abarca el control administrativo, el tamañodela organización, el número de niveles jerárquicos, la configuración de la jerarquía de los puestos, el grado de descentralización de la toma de decisiones, la especialización de funciones y tareas, el aspecto normativo, la formalización de procedimientos organizacionales y el grado de interdependencia entre diferentes subsistemas (La Torre, 2001; Bouroncle, 2010). La recompensa puede ser económica (sueldo, honorarios, propinas, bonos, etc.) o a través de estímulos psicosociales (reconocimiento, elogios, etc.) y la cooperación puede ser entendida como reciprocidad, sinergia funcional, espíritu de equipo, etc.

Para otros autores de habla hispana, las dimensiones del clima organizacional serían, según Isabel Pérez: la imagen gerencial, la calidad del ambiente, la integración organizacional y la vitalidad organizacional (Pérez, Maldonado y Bustamante, 2006). Casales y Sánchez (2004) basan sus estudios en una amplia variedad de elementos de los que depende el clima organizacional: los valores colectivos, las posibilidades de superación y desarrollo, los recursos materiales y el ambiente físico, la retribución material y moral, los estilos de dirección, el sentimiento de pertenencia, la motivación y el compromiso, la resolución de quejas y conflictos, las relaciones humanas, las relaciones jefe-subalternos, el control y las regulaciones, la estructura organizativa y el diseño del trabajo. Para Salgado, Remeseiro e Iglesias (1996), las dimensiones del clima organizacional son: la implicación, la cohesión, el apoyo, la autonomía, la organización, la presión, la claridad, el control, la innovación y la comodidad.

\section{Cuadro 1.}

Elementos del clima organizacional según autores de habla inglesa

\begin{tabular}{|c|c|c|c|c|c|c|c|c|c|c|c|c|c|c|}
\hline № & $\begin{array}{c}\text { ELEMENTOS } \\
\text { DEL CLIMA } \\
\text { ORGANIZACIONAL }\end{array}$ & $\frac{\mathrm{t}}{\mathrm{u}}$ & $\sum_{\bar{\Sigma}}^{\grave{d}}$ & $\sum_{\pi}^{5}$ & $\stackrel{\Xi}{3}$ & Е & 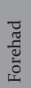 & 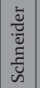 & $\frac{\Xi}{\frac{0}{n}}$ & 駕 & $\frac{\bar{\Xi}}{\overline{\tilde{E}}}$ & 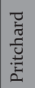 & 袋 & ङ \\
\hline o1 & Objetivos & $\mathrm{X}$ & & & $\mathrm{X}$ & & & & & & & & & 2 \\
\hline 02 & Cooperación & & $\mathrm{X}$ & $\mathrm{X}$ & & & $\mathrm{X}$ & & $\mathrm{X}$ & & & $\mathrm{X}$ & & 5 \\
\hline 03 & Liderazgo & $\mathrm{X}$ & $\mathrm{X}$ & & & & & $\mathrm{X}$ & $\mathrm{X}$ & & & & & 4 \\
\hline 04 & Toma de decisiones & $\mathrm{X}$ & & & & & & & & & & $\mathrm{X}$ & $\mathrm{X}$ & 3 \\
\hline 05 & Relaciones interpersonales & $\mathrm{X}$ & & & $\mathrm{X}$ & & $\mathrm{X}$ & $\mathrm{X}$ & $\mathrm{X}$ & & & $\mathrm{X}$ & $\mathrm{X}$ & 7 \\
\hline 06 & Motivación & $\mathrm{X}$ & & & & & $\mathrm{X}$ & & & & & $\mathrm{X}$ & $\mathrm{X}$ & 4 \\
\hline o7 & Control & $\mathrm{X}$ & & & & $\mathrm{X}$ & & & & & & & & 2 \\
\hline 08 & Conformidad & & $\mathrm{X}$ & & & & & & & & & & & 1 \\
\hline 09 & Responsabilidad & & $\mathrm{X}$ & & $\mathrm{X}$ & & & & & & & & & 2 \\
\hline 10 & Perfeccionamiento & $\mathrm{X}$ & & & & & & & $\mathrm{X}$ & & & & & 2 \\
\hline 11 & Confianza & & & $\mathrm{X}$ & & & $\mathrm{X}$ & $\mathrm{X}$ & & & & & & 3 \\
\hline 12 & Autonomía & & & & & & & & & $\mathrm{X}$ & $\mathrm{X}$ & $\mathrm{X}$ & & 3 \\
\hline 13 & Apoyo & & & & & & & & & $\mathrm{X}$ & $\mathrm{X}$ & $\mathrm{X}$ & & 3 \\
\hline 14 & Afecto & & & & & & & & & & $\mathrm{X}$ & & & 1 \\
\hline 15 & Recompensa & & $\mathrm{X}$ & $\mathrm{X}$ & $\mathrm{X}$ & & & & $\mathrm{X}$ & $\mathrm{X}$ & $\mathrm{X}$ & $\mathrm{X}$ & & 7 \\
\hline 16 & Conflicto & & & & $\mathrm{X}$ & & & & & & & $\mathrm{X}$ & & 2 \\
\hline 17 & Obstáculos & & & $\mathrm{X}$ & & & $\mathrm{X}$ & & & & & & & 2 \\
\hline 18 & \begin{tabular}{|l|} 
Procesos \\
\end{tabular} & $\mathrm{X}$ & & & $\mathrm{X}$ & & $\mathrm{X}$ & & $\mathrm{X}$ & & & & & 4 \\
\hline 19 & Organización & & $\mathrm{X}$ & & & $\mathrm{X}$ & & & & & & & & 2 \\
\hline 20 & Moral & & & & & & $\mathrm{X}$ & & $\mathrm{X}$ & & & & & 2 \\
\hline 21 & Estructura & & & $\mathrm{X}$ & $\mathrm{X}$ & & & $\mathrm{X}$ & & $\mathrm{X}$ & $\mathrm{X}$ & $\mathrm{X}$ & & 6 \\
\hline 22 & Normas & & $\mathrm{X}$ & & & & & & & & & & & 1 \\
\hline 23 & Riesgos y desafíos & & & $\mathrm{X}$ & $\mathrm{X}$ & & & & & & & & & 2 \\
\hline 25 & Cohesión & & & & & & & & $\mathrm{X}$ & & & & & 1 \\
\hline 26 & Comunicación & $\mathrm{X}$ & & & & & & & & & & & $\mathrm{X}$ & 2 \\
\hline 27 & Flexibilidad e innovación & & & & & & & & & & & $\mathrm{X}$ & $\mathrm{X}$ & 2 \\
\hline 28 & Nuevos empleados & & & & & & & $\mathrm{X}$ & & & & & & 1 \\
\hline
\end{tabular}


Estos elementos y/o dimensiones son utilizados para evaluar el clima organizacional y reflejan, a su vez, el enfoque dentro del cual se encuentra enmarcado dicho constructo.

\section{Evaluación del clima organizacional}

La evaluación o medición del clima organizacional es fundamental para cualquier empresa, pues permite 1) conocer las reacciones, disposiciones y valoraciones de los miembros de la organización; 2) disponer de información sobre las condiciones laborales; 3) potenciar los mecanismos de comunicación y relación; y 4) obtener una visión integradora de la organización (Guillén, Gala y Velázquez, 2000). De acuerdo con Gómez (2001), dentro de un contexto de cambio organizacional, el análisis y diagnóstico del clima organizacional permite 1) valorar las fuentes de conflicto, estrés o de insatisfacción; 2) iniciar y mantener un cambio que indique los elementos sobre los cuales deben dirigirse los esfuerzos de los miembros de la organización; 3) hacer el seguimiento del desarrollo de la organización y 4) prever los problemas que puedan surgir en el transcurso del tiempo.

Sin embargo, dado que determinantes del clima organizacional pueden ser tanto el ambiente físico como las características estructurales de la empresa, el ambiente social, las características personales y el comportamiento organizacional propiamente dicho (Salazar et al., 2009); Lassio (2003), indica que es necesario puntualizar los actores y las condiciones de la empresa antes de evaluar el clima organizacional. Agrega Lassio, siguiendo a James y Sells, que cinco son las áreas significativas para las personas en su entorno laboral: las características del rol, las características de la tarea, el comportamiento del líder, el trabajo en equipo y el entorno social organizacional. En ese sentido, diversos factores pueden influir en la medición del clima organizacional, como la posición de los empleados en la organización, la estructura de la organización, el tamaño, su grado de formalización, la personalidad del trabajador, los instrumentos de medición y los métodos o técnicas empleadas (Guillén, Gala y Velázquez, 200o). Precisamente, desde un punto de vista metodológico se distinguen tres modelos diferentes: a) la medida múltiple de atributos, b) la medida perceptiva de atributos, y c) y el modelo de filtro (Gómez, 2001).

Por otro lado, en la empresa puede haber varios climas diferentes. El clima de un área puede ser distinto de otra área. El clima total equivaldría a la medida de los climas reunidos en todos los departamentos o áreas, y es resultante de los micro-climas que lo componen (Bouroncle, 2010). Con respecto a los instrumentos de medición, las pruebas más utilizadas para medir el clima organizacional se encuentran en el Cuadro 2. 


\section{Cuadro 2.}

Instrumentos para evaluar el Clima

Organizacional

\begin{tabular}{|l|l|}
\hline Nombre del Instrumento & Autor \\
\hline Perfil Organizacional de Likert & Likert \\
\hline Inventario de Clima Psicológico & Gavin y Howe \\
\hline Índice de Clima Organizacional & Stern \\
\hline Cuestionario de Clima Organizacional & James y Sells \\
\hline Encuesta de Calidad de Empleo & Kahn \\
\hline Cuestionario Michigan de Evaluación Organizacional & Camman \\
\hline Escala de Ambientes de Trabajo & Moos \\
\hline Cuestionario Descriptivo del Clima Organizacional & Halpin y Crofts \\
\hline Cuestionario Descriptivo del Perfil del Clima Organizacional & Kettering \\
\hline Cuestionario de Salud Organizacional & Milles \\
\hline Escalas del Ambiente Universitario & Pace \\
\hline
\end{tabular}

A nivel de Latinoamérica, Colombia es el país donde se ha diseñado diversos test para medir el clima organizacional. Entre los más usados se tiene: el Instrumento de Medición de Clima Laboral en Organizaciones Colombianas de la Universidad del Rosario (IMCOC) y el Test de Clima Organizacional de la Universidad de los Andes de Bogotá (TECLA) (Gómez, 2001).

\section{Método}

\section{Muestra}

Se trabajó con una muestra de 354 trabajadores de ocho empresas de Arequipa, siendo 268 de ellos varones y 86 mujeres, con un rango de edad de entre 20 a 67 años. En el Cuadro 3 se aprecia la composición de la muestra por género, de acuerdo a la empresa en que laboran.
Cuadro 3.

Composición de la muestra

\begin{tabular}{|l|c|c|c|}
\hline Empresa & Varones & Mujeres & Total \\
\hline Empresa A & 20 & 6 & 26 \\
\hline Empresa B & 16 & 8 & 24 \\
\hline Empresa C & 20 & 11 & 31 \\
\hline Empresa D & 80 & 20 & 100 \\
\hline Empresa E & 58 & 2 & 60 \\
\hline Empresa F & 20 & 5 & 25 \\
\hline Empresa G & 18 & 26 & 44 \\
\hline Empresa H & 36 & 6 & 42 \\
\hline Total & 268 & 84 & 352 \\
\hline
\end{tabular}

Los participantes de este estudio fueron escogidos por la técnica de muestreo no probabilístico de grupos intactos. Con el fin de proteger la identidad de las empresas se les asignará en adelante una letra del alfabeto para diferenciarlas. De las empresas encuestadas, dos pertenecen al rubro de la educación (B de educación básica regular, $\mathrm{G}$ de educación superior); C es un estudio contable; A, D y $F$ son pequeñas empresas dedicadas a la producción de metales como ángulos ranurados, piezas para maquinarias, etc.; E y $\mathrm{H}$ brindan servicios para el cuidado del medio ambiente y sanidad pública.

\section{Instrumentos}

Para medir el clima organizacional se utilizó el Perfil Organizacional de Likert (POL) que consta de 25 preguntas con cinco alternativas de respuesta (siempre, casi siempre, a veces, casi nunca, nunca) que se puntúan de 5 a 1 y 
que ofrecen un puntaje total y puntajes parciales en función de seis dimensiones: flexibilidad, responsabilidad, estándares, reconocimiento, claridad y espíritu de equipo. A continuación se presenta una breve descripción de cada una de estas dimensiones:

Flexibilidad (Flex): valora la percepción que tienen los empleados con respecto a las limitaciones en el lugar de trabajo, es decir, el grado en el cual sienten que no hay reglas, procedimientos, políticas y prácticas innecesarias que interfieren con el logro de las tareas, y que las nuevas ideas sean fácilmente aceptadas.

Responsabilidad (Resp): es la percepción que tienen los empleados de tener autoridad delegada, es decir, valora el grado de libertad que tienen los trabajadores para ejecutar sus tareas y actividades asignadas por sus superiores, tomando la responsabilidad de los resultados obtenidos.

Estándares (Est): valora la percepción que tienen los empleados sobre el énfasis que pone la gerencia en el mejoramiento del desempeño, incluyendo el grado en el cual la gente siente que se fijan metas retadoras pero realizables.

Reconocimiento (Rec): se refiere al grado en que los empleados perciben que están siendo reconocidos y premiados por realizar un buen trabajo, y que dicho reconocimiento esté directa y diferencialmente relacionado con los niveles de desempeño.
Claridad (Clar): es la percepción de que los trabajadores saben lo que se espera de ellos y que entienden cómo dichas expectativas se relacionan con las metas y objetivos más globales de la organización.

Espíritu de Equipo (E Equ): refiere la percepción de los trabajadores del prestigio de la empresa, valora el orgullo que tienen las personas respecto de la organización en que laboran. Indica el grado de confianza de que todos están trabajando hacia un objetivo común.

Este instrumento, obedece a un concepto del clima organizacional como percepción del medio interno de la empresa, vinculándolo a su vez con otras variables organizacionales. Además, esta prueba permite establecer un perfil sobre las seis dimensiones del clima organizacional anteriormente señaladas, de modo que es posibledeterminarel grado deflexibilidad, responsabilidad, perfeccionamiento, reconocimiento, claridad y cooperación que existe en la empresa. Estos niveles se valoran en cinco niveles según los puntajes obtenidos. De o a 5 el nivel de clima organizacional es deficiente, de 6 a 10 puntos el clima es moderado bajo, de 11 a 15 el clima es moderado, de 16 a 20 se considera moderado alto y de 21 a 25 el nivel del clima organizacional sería elevado. Este instrumento cuenta además con un nivel de confiabilidad de o.88.

\section{Procedimientos}

Para la valoración del clima organizacional en cada una de las empresas, se solicitaron los permisos correspondientes ante los 
directivos y jefes de recursos humanos de cada una de estas instituciones y se coordinaron las condiciones en que se efectuarían las evaluaciones de los trabajadores. Se procedió a evaluar a los trabajadores en sus horarios de descanso de manera colectiva y en ambientes cerrados. Las evaluaciones fueron realizadas por el autor y un grupo de colaboradores que recibieron entrenamiento para aplicar correctamente el instrumento de medición. Tras la aplicación del mismo, se entregaron los resultados a los directivos de cada empresa, con una breve lista de recomendaciones y sugerencias para mejorar las condiciones laborales de los trabajadores. La información fue recolectada entre los meses de enero de 2010 y marzo de 2011.

\section{Resultados}

Los datos fueron procesados descriptivamente, mediante el análisis de frecuencias y porcentajes. Cabe mencionar que los datos se procesaron inicialmente en función de las diversas divisiones y departamentos que presentan las empresas de las que se han tomado las muestras, pero para efectos de normalizar los datos y de compararlos de manera uniforme, se han tomado los valores por dimensión y los totales como se aprecia en el Cuadro 4, sin considerar los puntajes obtenidos por divisiones dentro de las organizaciones.
Cuadro 4.

Dimensiones del Clima Organizacional en las empresas evaluadas

\begin{tabular}{|l|c|c|c|c|c|c|c|}
\hline \multirow{2}{*}{ Empresas } & \multicolumn{7}{|c|}{ Dimensiones del Clima Organizacional } \\
\cline { 2 - 8 } & Flex & Resp & Est & Rec & Clar & E Equ & Total \\
\hline Empresa A & 15,80 & 17,30 & 15,00 & 17,37 & 17,37 & 18,60 & 16,90 \\
\hline Empresa B & 15,50 & 18,87 & 19,00 & 15,37 & 18,37 & 20,00 & 17,85 \\
\hline Empresa C & 19,19 & 21,01 & 20,95 & 19,87 & 19,58 & 21,37 & 20,32 \\
\hline Empresa D & 15,78 & 21,62 & 20,08 & 17,21 & 23,50 & 21,85 & 20,00 \\
\hline Empresa E & 18,53 & 17,47 & 11,66 & 8,50 & 19,66 & 13,75 & 15,52 \\
\hline Empresa F & 16,30 & 16,32 & 16,21 & 14,41 & 18,37 & 16,90 & 16,41 \\
\hline Empresa G & 17,36 & 15,37 & 17,98 & 12,12 & 16,52 & 16,86 & 16,04 \\
\hline Empresa H & 16,97 & 17,63 & 17,33 & 10,37 & 20,02 & 16,26 & 16,42 \\
\hline Total & 16,94 & 17,95 & 17,29 & 12,67 & 19,53 & 17,36 & 16,80 \\
\hline
\end{tabular}

En la Figura 1 se observa que, de las ocho empresas, la dimensión del clima organizacional que tiene las puntuaciones más altas es la de claridad $(19,53)$, seguida de responsabilidad (17,95), espíritu de equipo $(17,36)$, estándares $(17,29)$, flexibilidad $(16,94)$; en último lugar, se ubica la dimensión de reconocimiento $(12,67)$. También se observa que las empresas C y D tienen el perfil más elevado, mientras que lo opuesto ocurre con las empresas E y G.

\section{Figura 1.}

Polígono de Frecuencias de las dimensiones del Clima Organizacional

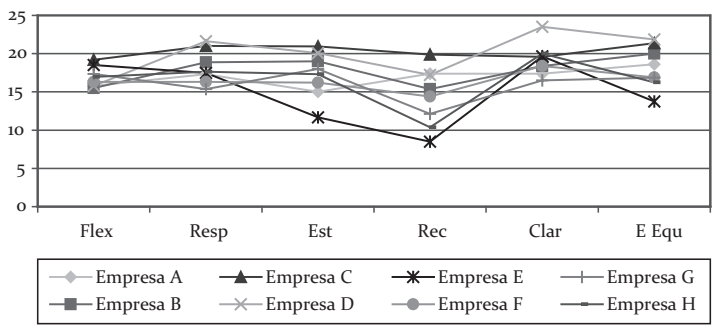


Las empresas C $(19,19)$ y E $(18,53)$ tienen niveles moderados altos de flexibilidad, mientras que las empresas B $(15,15)$ y D $(15,78)$ presentan los niveles más bajos (moderados bajos). En la dimensión de responsabilidad, las empresas D $(21,62)$ y $C(21,01)$ tienen el nivel más elevado. Mientras que las empresas F $(16,32)$ y $G$ $(15,37)$ alcanzan un nivel moderado alto y moderado, respectivamente. Con respecto a la dimensión de estándares, las empresas C $(20,95)$ y D $(20,08)$ presentan los niveles más altos. Las empresas E $(11,66)$ y A $(15)$ se ubican muy por debajo de las demás en esta dimensión (con niveles moderados bajos). En cuanto a la dimensión de reconocimiento, las empresas C $(19,87)$ y A $(17,37)$ cuentan con los niveles más elevados (moderado alto), pero las empresas E $(8,5)$ y $\mathrm{H}(10,37)$ se ubican en niveles moderados bajos y moderados, respectivamente.

El análisis de la dimensión claridad, posiciona a las empresas $\mathrm{D}(23,5)$ y $\mathrm{H}(20,02)$ como las empresas con mayor nivel en esta dimensión (alto para $\mathrm{D}$ y H), mientras que las empresas $G(16,52)$ y A $(17,37)$ alcanzan los niveles moderados bajos. Finalmente, en la dimensión espíritu de equipo, las empresas D $(21,85)$ y C $(21,37)$ alcanzan nuevamente los niveles más elevados, en tanto que las empresas $\mathrm{E}(13,75)$ y $\mathrm{H}$ $(16,26)$ obtienen puntuaciones moderadas y moderadas bajas.

Al revisar los puntajes obtenidos de las ocho empresas, se tiene que las empresas C $(20,32)$ y D $(20,00)$ han obtenido los valores más altos, mientras que la empresa E $(15,32)$ registra los puntajes más bajos (niveles moderados). Las empresas B $(17,85)$, A $(16,90), \quad$ F $(16,41)$ y $G(16,04)$ presentan valores moderados altos. A pesar de las deficiencias en las dimensiones de reconocimiento y flexibilidad, el nivel del clima organizacional de las empresas evaluadas es moderado alto $(16,80)$.

\section{Figura 2.}

Gráfico de barras del puntaje total de Clima Organizacional

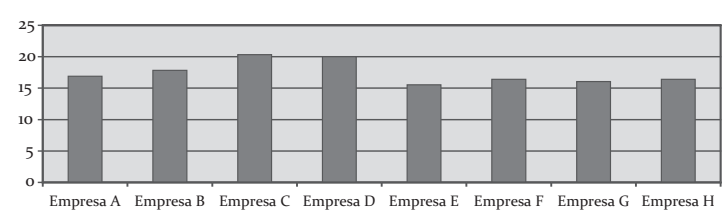

\section{Discusión}

En los últimos años, las investigaciones sobre clima organizacional se han convertido en una herramienta estratégica que favorece la intervención de los gerentes en los puntos que consideren críticos, yestablecerasí propuestas de mejoras oportunas que permitan obtener nuevos logros, corrigiendo a tiempo aquellos factores que afectan la producción y el rendimiento de los trabajadores (Smith, 2006). Consecuentemente, la coordinación entre los miembros del grupo tiende a estar positivamente relacionada con el desempeño (Ripoll y Orengo, 2004). Por ello, el clima organizacional es fundamental para las organizaciones, ya que si los trabajadores no mantienen buenas las relaciones interpersonales, no cooperan entre sí y no asumen como propias la visión y la misión de la empresa, difícilmente podrán contribuir con el desarrollo organizacional de su centro de labores. 
Por otro lado, las condiciones de trabajo como las condiciones del empleo (contrato), condiciones ambientales (ambiente físico, variables espacio-geográficas, aspectos del diseño espacial y arquitectónico), condiciones de seguridad, características de las tareas, procesos de trabajo, condiciones sociales, etc. (Guillén, Gala y Velázquez, 200o) deben estimular el involucramiento del trabajador para con las metas de la empresa. El clima organizacional es un constructo que se ubica en medio de todas estas variables, ya que si bien depende de las influencias del entorno laboral, se forma también de las creencias y percepciones del trabajador. En ese sentido, existen relaciones entreel clima organizacional y la personalidad de los trabajadores, de modo que las condiciones objetivas de la organización se encuentran mediadas por la percepción que tiene el empleado de su entorno laboral. De hecho, las personas actúan y reaccionan a sus condiciones laborales, no por lo que estas condiciones son, sino a partir de la imagen que de ellas se forman (Casales y Sánchez, 2004).

En el presente estudio se ha evaluado el clima organizacional de ocho empresas arequipeñas. Algunas pequeñas y otras medianas o grandes, en diversos rubros de producción y prestación de servicios. Dado que las empresas no se enmarcan dentro de un mismo tipo de mercado ni comparten los bienes y servicios que ofertan, este hecho constituye una limitación que debe tomarse en cuenta a la hora de generalizar los resultados.

Podemos ver que la flexibilidad ha sido una dimensión que ha alcanzado valores moderados altos. Con respecto a la responsabilidad, se tienen valores moderados altos y altos en esta dimensión. De hecho, esta dimensión es la segunda más elevada después de claridad. La responsabilidad se relaciona con las demandas de los trabajadores y la autonomía que poseen en su puesto de trabajo. Precisamente, la falta de autonomía en el trabajo se asocia, según Boada, de Diego y Agulló (2004), con las jaquecas y otras manifestaciones psicosomáticas. De modo que es posible que los trabajadores de la empresa G (institución educativa superior) presenten síntomas psicofisiológicos, puesto que tienen los niveles más bajos de responsabilidad. Además, es probable que en esta empresa exista una excesiva burocratización y relaciones tanto verticales como centralizadas. Sin embrago, en términos generales, parece ser que a los trabajadores que conforman la muestra de nuestro estudio, se les otorga libertad en el trabajo dentro del marco de sus funciones establecidas. Ello se desprende, debido a que tanto las dimensiones de claridad y flexibilidad son las más elevadas.

En la dimensión de estándares las empresas C y D han obtenido los puntajes más elevados, es decir que en estas organizaciones existe un interés por el perfeccionamiento continuo, lo cual es coherente con el hecho de que ambas empresas presentan el perfil más alto de clima organizacional, incluyendo las dimensiones de reconocimiento (empresa C), claridad (empresa D) y espíritu de equipo (empresa D). Ahora bien, aunque es importante que las empresas exijan un mejor desempeño de parte de sus colaboradores, algunos estudios sugieren 
que un clima organizacional basado sólo en la innovación potencia el cansancio emocional y la despersonalización (Boada, de Diego y Agulló, 2004). De modo que, de acuerdo a los resultados obtenidos, deben considerarse con cuidado las exigencias que se imponen en las empresas.

La dimensión de reconocimiento es la que menos puntaje obtiene en comparación con las otras dimensiones, alcanzando niveles moderados bajos o moderados en la mayoría de los casos. Esto sugiere que las empresas arequipeñas, en general, no estimulan ni recompensan a sus empleados, lo cual debe considerarse con urgencia, ya que las recompensas tienen injerencia en la motivación del trabajador y su satisfacción. En ese sentido, el clima organizacional afecta la conducta de los trabajadores a través de los estímulos del ambiente, las limitaciones percibidas y las recompensas que reciben (Guillén, Gala y Velázquez, 200o). Estas recompensas, empero, no necesariamente deben ser económicas sino que puede orientarse a mejorar la moral del trabajador (Salvatierra, 200o), por tanto es fácil implementar medidas que reconozcan la labor de los trabajadores, sino económicamente, al menos moralmente.

La claridad como dimensión que designa el grado de conocimiento que tienen los trabajadores sobre su propio trabajo y los objetivos de la empresa en la que laboran, hasta el punto de involucrarse con ellos, ha obtenido el puntaje más elevado de todas las escalas del perfil organizacional de Likert (niveles altos y moderados altos). El espíritu de equipo es la dimensión que se ubica en tercer lugar, después de la responsabilidad. La dimensión de espíritu de equipo ha sido relacionada con los diversos elementos del clima organizacional como las relaciones interpersonales y el apoyo. En ese sentido, un clima basado en el apoyo y consecución de metas incrementa la realización personal (Boada, de Diego y Agulló, 2004). De hecho, estudios anteriores han probado la existencia de correlaciones significativas entre el apoyo del grupo de trabajo, los climas organizacionales positivos y la satisfacción laboral de los empleados (Romero, 2001).

Cierto es, empero, que las relaciones interpersonales son fundamentales para el constructo de clima organizacional. En ese sentido, los resultados de Ripoll y Orengo (2004), sugieren que el tipo de interacción entre los miembros del grupo influye en la cohesión que se alcanza en las tareas asignadas. En un estudio del clima organizacional realizado por Romero, en 917 trabajadores, se encontró que la escala más elevada fue la de compañerismo. Además, el clima organizacional correlaciona positivamente con el bienestar psicológico y negativamente con la depresión. Asimismo, las dimensiones de apoyo, metas e innovación correlacionaron positivamente con la satisfacción laboral (Romero, 2001).

Ahora bien, en nuestro estudio las dimensiones del clima organizacional que alcanzan los niveles más elevados fueron: claridad, responsabilidad y espíritu de equipo. Mientras que León (200o) identifica el control, el conocimiento de los objetivos corporativos y el deseo de participar en la toma de decisiones como las dimensiones 
del clima organizacional que actúan como fortalezas en las empresas. Cabe notar que estos elementos se corresponden con las dimensiones de claridad y responsabilidad de Likert, que son los más elevados en nuestra investigación. En otro estudio que se efectuó en una muestra de 130 trabajadores, se encontró que las fortalezas eran la satisfacción con el trabajo de sus miembros y la percepción de posibilidades de superación (Casales y Sánchez, 2004). Estos autores también han encontrado que a mayor grado de instrucción, existe una mayor insatisfacción laboral, y que los trabajadores más antiguos se sienten más satisfechos que los jóvenes, tienen mayor compromiso con las metas de la empresa y mantienen mejores relaciones con sus jefes.

Por otro lado, las debilidades de las empresas que se han identificado a través de la evaluación del clima organizacional son la falta de cooperación y comunicación, la falta de políticas claras de reconocimiento, la toma de decisiones concentrada, el bajo compromiso, la desmotivación y la inseguridad generalizada (León, 2000). En nuestra investigación, han sido el reconocimiento y la flexibilidad las dimensiones más deficitarias. De este hecho se desprende que los trabajadores perciben que se les exige más de lo que se les da, y que no cuentan con el apoyo de los directivos para conseguir las metas propuestas. Sin embargo, a pesar de las deficiencias en las dimensiones de reconocimiento y flexibilidad, el nivel del clima organizacional de las empresas evaluadas es moderado alto $(16,80)$.
Se concluye que, en las empresas evaluadas, los trabajadores perciben que se les exige un desempeño orientado a la calidad y el perfeccionamiento, pero como contraparte, no se les brinda el apoyo ni las recompensas necesarias para cumplir con los objetivos organizacionales. Asimismo, ante este panorama, los trabajadores parecen tomar las relaciones interpersonales con sus compañeros, como fuentes de apoyo social que permiten suplir la falta de apoyo institucional. Ello sumado a la claridad de sus funciones, constituyen las fortalezas de las organizaciones de este estudio. Por lo cual se infiere que los trabajadores tienen autonomía en el trabajo pero sólo dentro del marco de sus funciones asignadas.

Finalmente, podemos decir, al igual que Gómez (2001), que el clima organizacional refleja los valores, actitudes y las creencias de los miembros de una organización. Pero también es necesario comprender que el ambiente de trabajoy el clima organizacional son fenómenos socialmente construidos, que surgen de las interacciones individuogrupo-condiciones de trabajo. Por tanto, el clima organizacional es el resultado de una gestión bien concebida y dirigida (Pérez, Maldonado y Bustamante, 2006), de modo que los gerentes y directivos de las empresas tienen sobre sus hombros la vital tarea de promover un clima organizacional lo más satisfactorio y positivo posible para los trabajadores.

\section{Recomendaciones}

Se recomienda implementar estrategias que reconozcan la labor de los empleados, 
como cartas de felicitación, la designación del empleado del mes o la oferta de bonos económicos a los mejores empleados o los que registran mayores niveles de desempeño, innovación y productividad. Además, dados los niveles presentes de clima organizacional, se sugiere realizar evaluaciones de estrés laboral, síndrome de burnout y satisfacción laboral, entre otras valoraciones, para detectar posibles factores de riesgo psicosocial que se relacionan con las dimensiones del clima organizacional, y así prevenir enfermedades ocupacionales y accidentes dentro de la organización, en especial aquellas que se ubican dentro del sector industrial.

\section{Referencias}

Alcover, C. M. (2003). Cultura y clima organizacional. En Gil, F. y Alcover, C. M. (Comps.), "Introducción a la Psicología de las Organizaciones" (p. 387-414). Madrid: Alianza Editorial.

Arias, M. (2007). Factores del clima organizacional influyentes en la satisfacción laboral de enfermería, concerniente a los cuidados intensivos neonatales del Hospital Nacional de Niños, 2004. "Enfermería en Costa Rica", 28(1), 12-19.

Arias, W. L. (2005). "Psicólogos”. Arequipa: Faraday.

Atalaya, M. (2001). El estrés laboral y su influencia en el trabajo. Industrial Data, 4(2), 25-36.

Barrón, J.; Soler, C. E. y Bongiovanni, C. (2005). "Influencia del clima organizacional en el estrés laboral de las PYMES de Río Cuarto”. Trabajo presentado en las XVI Jornadas de Investigación y Trabajo Científico de la Facultad de Ciencias Económicas de la Universidad Nacional de Río Cuarto.

Boada, J.; de Diego, R.; Agulló, E. (2004). El burnout $y$ las manifestaciones psicosomáticas como consecuentes del clima organizacional y de la motivación laboral. "Psicothema", 16(1), 125-131.

Bouroncle. S. (2010). "Clima del trabajo en las organizaciones.” Arequipa: UNSA.

Casales, J. C. y Sánchez, I. (2004). Variables organizacionales que afectan el funcionamiento de una empresa de proyectos. "Revista Cubana de Psicología”, 21(3), 189-205.

Chiavenato, I. (2002). "Administración de recursos humanos”. México: McGraw-Hill.

Chiavenato, I. (2009). "Gestión del talento humano". México: McGraw-Hill.

Gómez, A. M.; Sánchez, J. C. y Alonso, E. (2005). Cultura organizacional. En Palaí, F. J. (Comp.), "Psicología de la organización” (p. 217-244). Madrid: Pearson Prentice Hall.

Gómez, C. A. (2001). Diseño, construcción y validación de un instrumento que evalúa clima organizacional en empresas colombianas, desde la teoría de respuesta al ítem. "Acta Colombiana de Psicología", 11(4), 97-113.

Guillén, C.; Gala, F, y Velázquez, R. (200o). Clima organizacional. En Guillén, C. y Guill, R. (comps.), "Psicología del trabajo para relaciones laborales” (p. 164-179). Madrid: McGraw-Hill.

Lassio, M. V. (2003). Clima y su evolución hacia un concepto estratégico. "Revista Latinoamericana de Administración”, 30, 87-95. 
La Torre. H. (2001). "Psicología Salazar, J. G.; Guerrero, J. C.; Machado, Y. organizacional”. Arequipa: UNSA.

León, A. (2000). Clima organizacional. Antesala del aseguramiento de la calidad. "Ingeniería y desarrollo", 8, 25-32.

B. y Cadeño, R. (2009). Clima y cultura organizacional: dos componentes esenciales en la productividad laboral. “ACIMED”, 20(4), 67-75.

Pérez, I.; Maldonado, M. y Bustamante, S. (2006). Clima organizacional y gerencia: Inductores del cambio organizacional. “Investigación y postgrado", 21(2), 231-248.

Portugal, L. (2000). "Psicología social". Arequipa: UNSA.

Ripoll, P. y Orengo, V. (2004). La influencia de los procesos de interacción grupal y el medio de comunicación sobre la eficacia de los grupos de trabajo. "Revista Latinoamericana de Psicología”, 36(2), 195-208.

Robbins. S. (1999). "Comportamiento organizacional”. México: Prentice Hall.

Romero, J. M. (2001). Clima laboral y bienestar psicológico en una empresa pública. "Archivos de Prevención de Riesgos Laborales", 4(1), 17-23.

Salgado, J. F.; Remeseiro, C. e Iglesias, M. (1996). Clima organizacional y satisfacción laboral en una PYME. "Psicothema", 8(2), 329-335.

Salvatierra. B. (2000). “Orientación psicológica laboral”. Arequipa: UNSA.

Sandoval, M. C. (2004). Concepto y dimensiones del clima organizacional. "Hitos de Ciencias Económico Administrativas", 10(27), 78-82.

Smith, H. (2006). Evaluación de los procesos organizacionales como estrategia de intervención para el cambio organizacional. "Multiciencia”, 6(1), 1-16. Vega, D.; Arévalo, A.; Sandoval, J.; Aguilar, M. C. y Giraldo, J. (2006). Panorama sobre los estudios de clima organizacional en Bogotá, Colombia (1994-2005). "Revista Diversitas", 2(2), 329-349. 